Supporting Information for

\title{
Colloidal Bimetallic Nanorings for Strong Plasmon Exciton
}

\section{Coupling}

C. Meric Guvenc, ${ }^{\dagger}$ Fadime Mert Balci, ${ }^{\ddagger}$ Sema Sarisozen,,${ }^{\S}$ Nahit Polat,,${ }^{\ddagger}$ and Sinan Balci ${ }^{*}$

$\dagger$ Department of Materials Science and Engineering, Izmir Institute of Technology, 35430

Izmir, Turkey

tDepartment of Photonics, Izmir Institute of Technology, 35430 Izmir, Turkey

$\S$ Department of Chemistry, Izmir Institute of Technology, 35430 Izmir, Turkey

*E-mail: sinanbalci@iyte.edu.tr

\section{Table of Contents:}

Figure S1. Large area STEM image of plasmonic nanorings.

Figure S2. EDX analysis of silver-gold nanorings.

Figure S3. EDX elemental analysis results of silver-gold nanorings.

Figure S4. Absorbance spectrum of J-aggregate.

Figure S5. Nanoprism to nanodisk conversion by heating.

Figure S6. Tuning color of silver nanodisk by galvanic replacement reaction.

Figure S7. Large area STEM image of silver nanodisks.

Figure S8. Extinction spectra of nanodisk shaped plasmonic and plexcitonic nanoparticles.

Numerical calculations. Numerical calculations of nanoring shaped plasmonic and plexcitonic nanoparticles.

Figure S9. Extinction spectra of nanodisk shaped plasmonic and plexcitonic nanoparticles.

Figure S10. Imaginary and real parts of the dielectric function of Lorentz Oscillator.

\section{References}




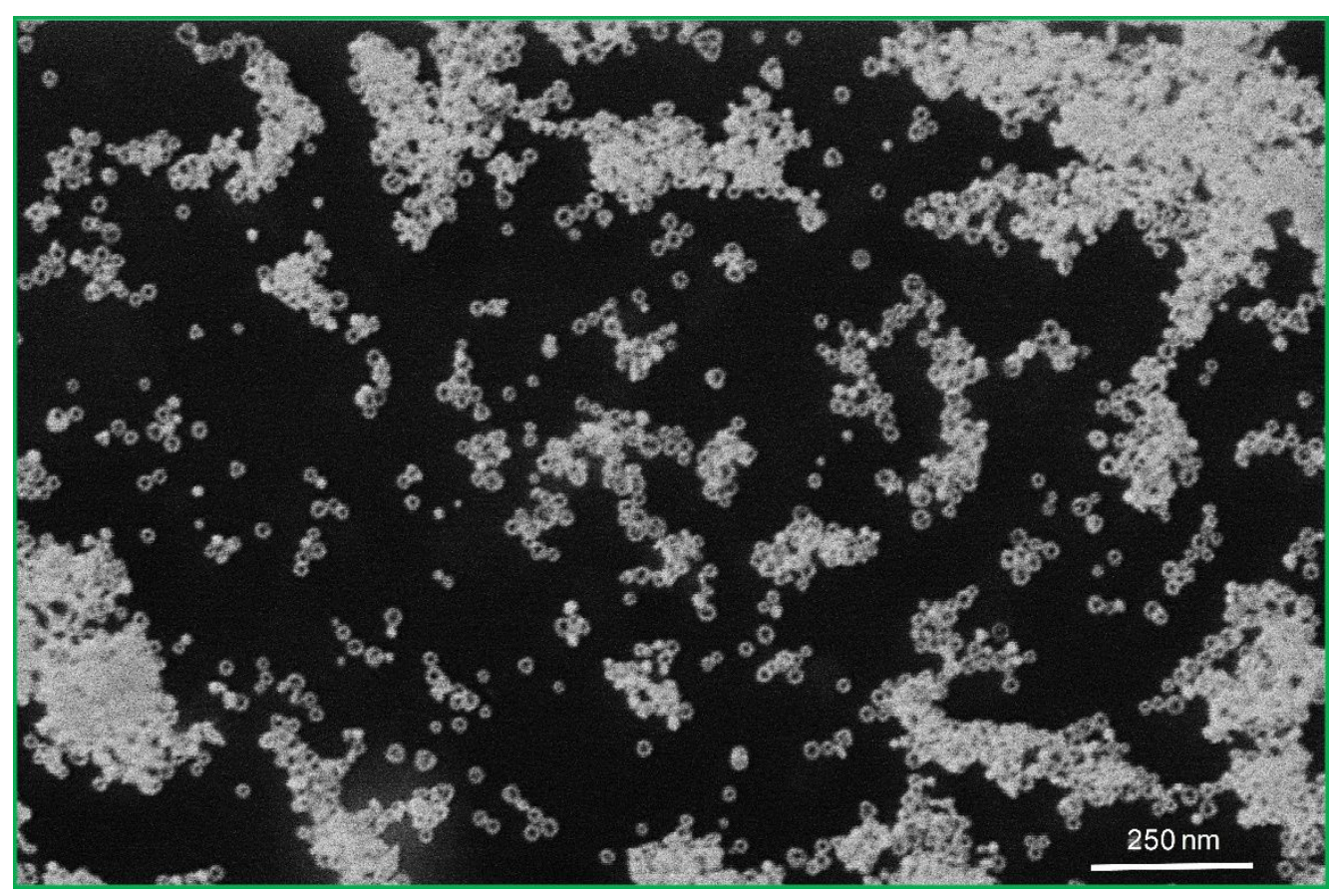

Figure S1. Plasmonic nanorings. Large area STEM image of nanorings synthesized by adding excess amount of gold ions into silver nanodisk colloid. Addition of trace amount of gold ions into silver nanodisk colloid generates nanoholes on the nanodisks. Most of the bimetallic nanorings are in circular shaped. The inner dimater of the nanoring can be controlled by the amount of gold ions in galvanic displacement reaction. In order to syntehsize bimetallic nanorings, firstly, seed mediated silver nanoprisms were colloidally syntehsized and than the nanoprisms were converted to nanodisks at high temperature. ${ }^{1}$ Addition of gold ions drilled holes on the plasmonic nanodisks. Note that small nanoholes poseses high intensity eelctric field, which is called plasmonic hot spot. Plasmonic hot spots can be sued to enhance lightmatter interaction at nanoscale dimension. ${ }^{2}$ 

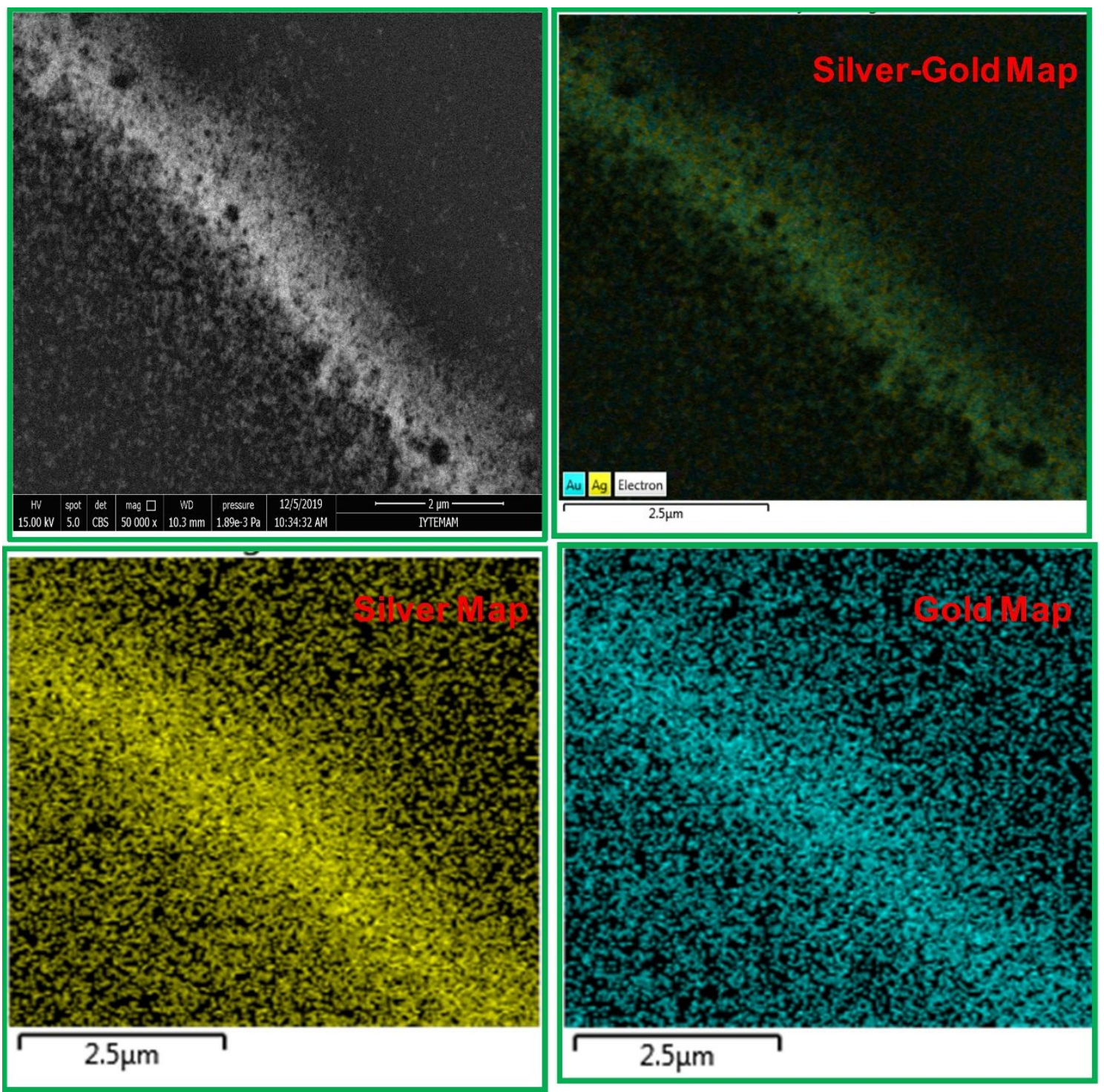

Figure S2. EDX analysis of silver-gold nanorings. The EDX maps clearly shows presence of gold and silver in nanorings. 

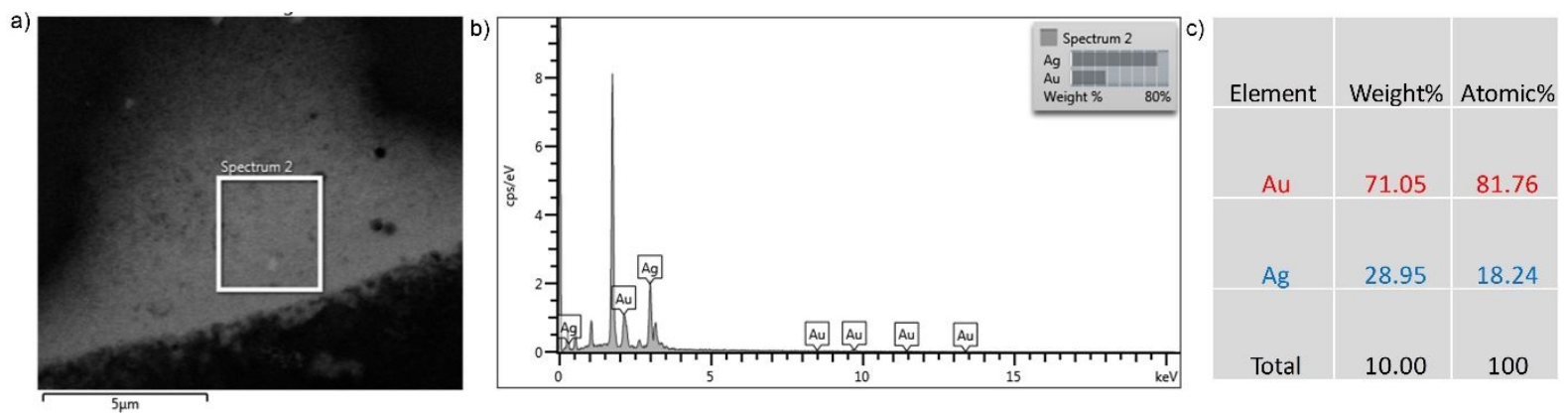

Figure S3. EDX elemental analysis results of silver-gold nanorings. (a) Large area SEM image of the nanorings. (b) EDX spectrum of the enclosed area indicated in (a). (c) Elemental composition from EDX analysis. The table shows the elements detected by EDX analysis from gold-silver nanorings.

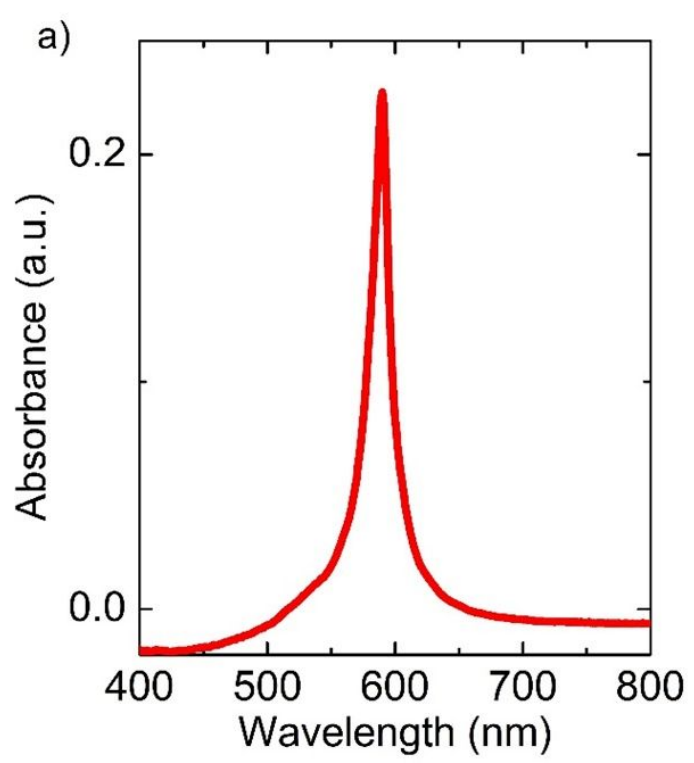

b)<smiles>CCN1C(=CC=Cc2n(CC)c3cc(Cl)c(Cl)cc3[n+]2CCCCS(=O)(=O)[O-])N(CCCC[S-]([O])([O-])[O-])c2cc(Cl)c(Cl)cc21</smiles>

Figure S4. (a) Absorbance spectrum of J-aggregated TDBC cyanine dye in aqueous solution. (b) Chemical structure of a TDBC molecule. The sharp absorbance of aggregated dye is at around $584 \mathrm{~nm}$. At high concentration, individual dye molecules self-assemble end-to-end and J-aggregates are formed. ${ }^{3} \mathrm{~J}$-aggregates have red-shifted absorbance peak with respect to the monomer absorbance peak. 

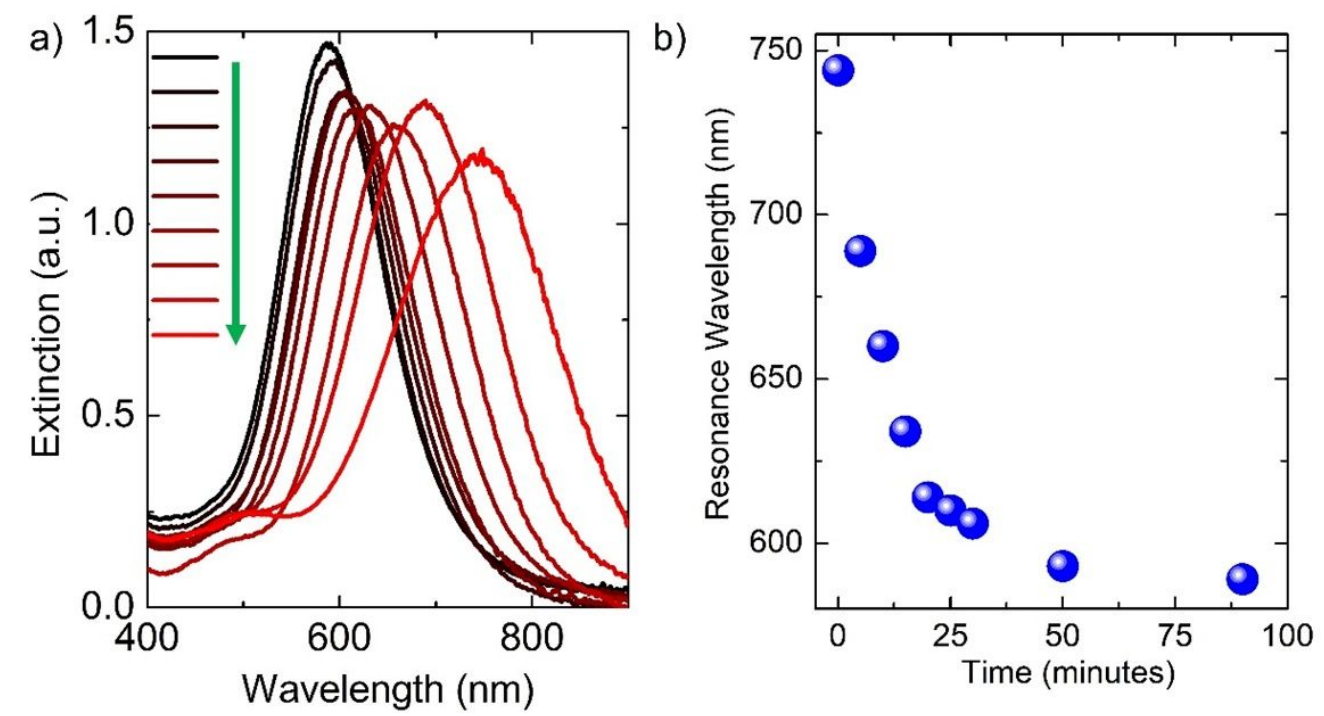

Figure S5. Shape conversion of nanoprisms to nanodisks through heating. (a) Extinction spectra of the silver nanoprism colloid during heating. The spectra shifts shorter wavelengths due to the shape conversion from nanoprism to nanodisk. The green arrow indicates the direction of temperature increase during the heating process. (b) Corresponding plasmon resonance wavelength as a function of heating time. Heating was performed in the extinction spectra from black colored curves to red colored curves.

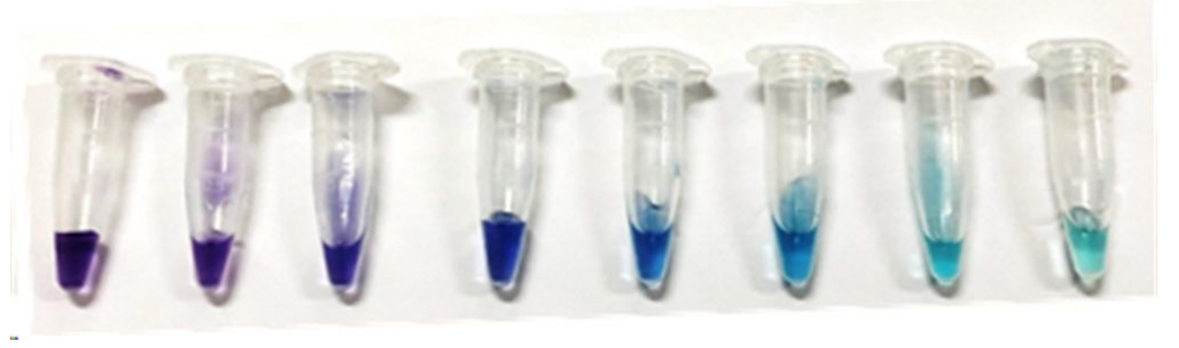

Figure S6. Tuning color of silver nanodisk by galvanic replacement reaction. The color of the colloid changes from dark purple to bright blue as the amount of $\mathrm{HAuCl}_{4}$ increases. The extinction spectra shows red shifted plasmon resonance wavelengths. 


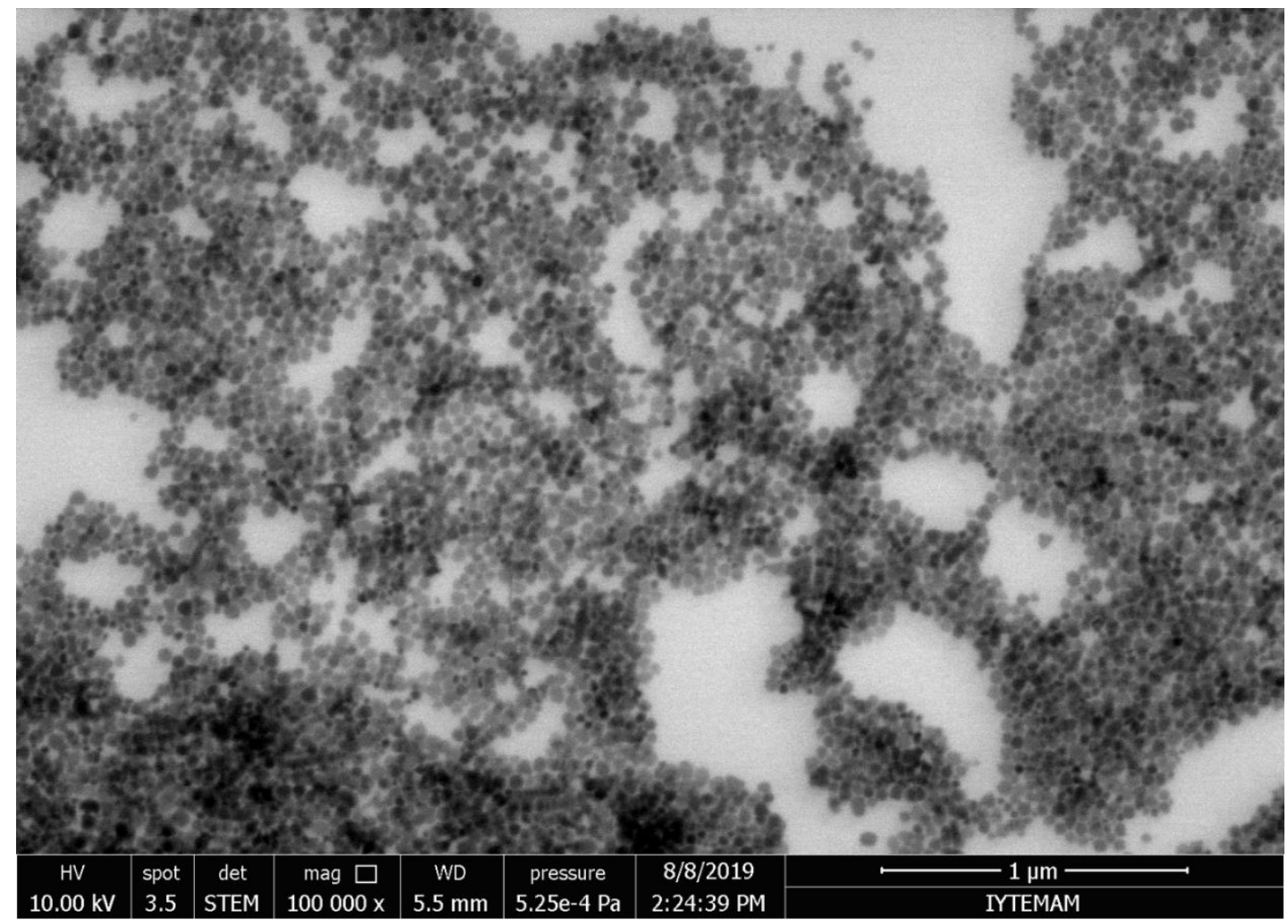

Figure S7. Large area STEM image of silver nanodisks synthesized by heating silver nanoprism at high temperature. Most of the nanoprisms were converted to nanodisks. 

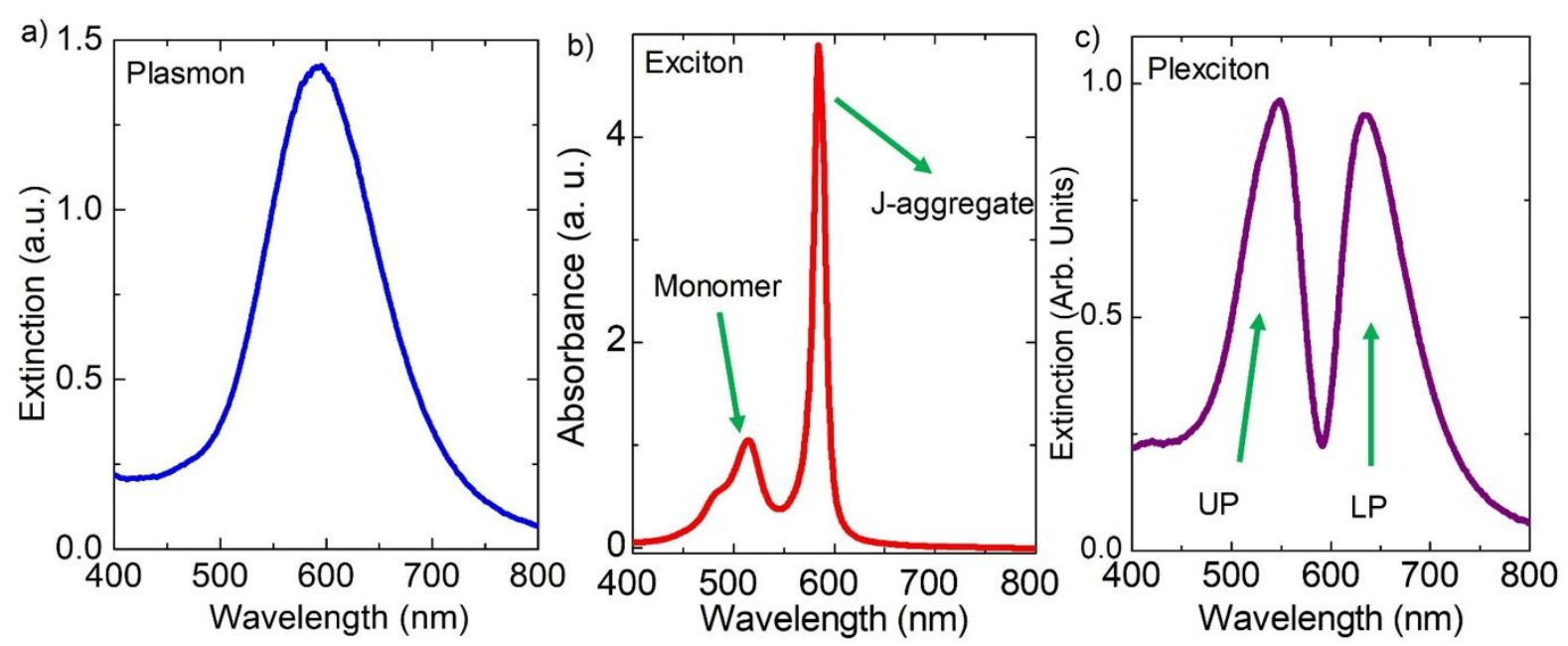

Figure S8. Nanodisk shaped plexcitonic nanoparticles. (a) Extinction spectrum of nanodisk shaped plasmonic nanoparticles. Localized SPP resonance wavelength of the nanodisks are around $600 \mathrm{~nm}$. The nanodisks were obtained by heating nanoprisms shaped plasmonic nanoparticles at high temperature. (b) Absorbance spectrum of J-aggregate dye. The broad peak at round $520 \mathrm{~nm}$ corresponds to absorbance of monomer dye molecules. The sharp resonance peak at around $585 \mathrm{~nm}$ is absorbance of aggregates dye molecules. (c) The dye molecules self assemble of plasmonic nanodisks and transparency deep is observed at around $585 \mathrm{~nm}$. The UP and LP correspond to upper polariton and lower polariton branches, respectively. Note that the uncoupled dye molecules were completely removed by cantrifugation before the extinction spectrum was taken as discussed in detail in our previous works. ${ }^{4}$ 


\section{Numerical calculations}

In this study, the experimental results were confirmed by numerical calculations. In the numerical calculations, the metallic nanodisk with a nanohole in the center was suspended in air. The Frenkel exciton was assumed to be Lorentzian and expressed as $\varepsilon(\omega)=\varepsilon_{\infty}+f_{0}\left(\omega_{0}{ }^{2} /\left(\omega_{0}{ }^{2}-\right.\right.$ $\left.\left.\omega^{2}-i \gamma_{0} \omega\right)\right)$ in which the resonance wavelength of the oscillator was set to $587 \mathrm{~nm}(2.11 \mathrm{eV})$, the width of the exciton resonance $\left(\gamma_{0}\right)$ was set to around $32 \mathrm{meV}$. The background index, $\varepsilon_{\infty}$, was set to 2.1. The oscillator strength of the Lorentz oscillator $\left(f_{0}\right)$ was set to vary from 0 to 0.1 . A very narrow dip in the extinction spectra is a very clear indication of strong coupling between an exciton and a plasmon. However, anticrossing in the the dispersion curve of the coupled system has to be obtained in order to strong coupling. In our coupled system, we are able to tune plasmon resonance wavelength from $400 \mathrm{~nm}$ to $1100 \mathrm{~nm}^{5}$ and thus we can study interaction of plasmon and exciton in broad range of wavelengths. On the other hand, the exciton resonance is fixed to $2.11 \mathrm{eV}$. At zero detuning in the dispersion curve, Rabi splitting energy reflecting coupling strength between exciton and plasmon is found. Note that in the strong coupling plasmon and exciton reversibly exchange energy. It should be pointed out here that Rabi splitting energy, $\hbar \Omega \sim\left(f_{0}\right)^{1 / 2}$, depends on oscillator strength, $f_{0}$, of the exciton. The nanoholes shown in this work localize electromagnetic waves in very small region, $\sim 6 \mathrm{~nm}$, can be considered as plasmonic nanocavities. Since the coupling strength $(g), \hbar \Omega=2 g \sim 1 /(V)^{1 / 2}$, is proportional to the effective mode volume of the cavity, $V$, we expect to see enhancement in light matter interaction in plasmonic cavities. Therefore, they can be used to study light matter interaction at nanoscale dimension, for example, single molecule strong coupling at room temperature. ${ }^{6}$ 

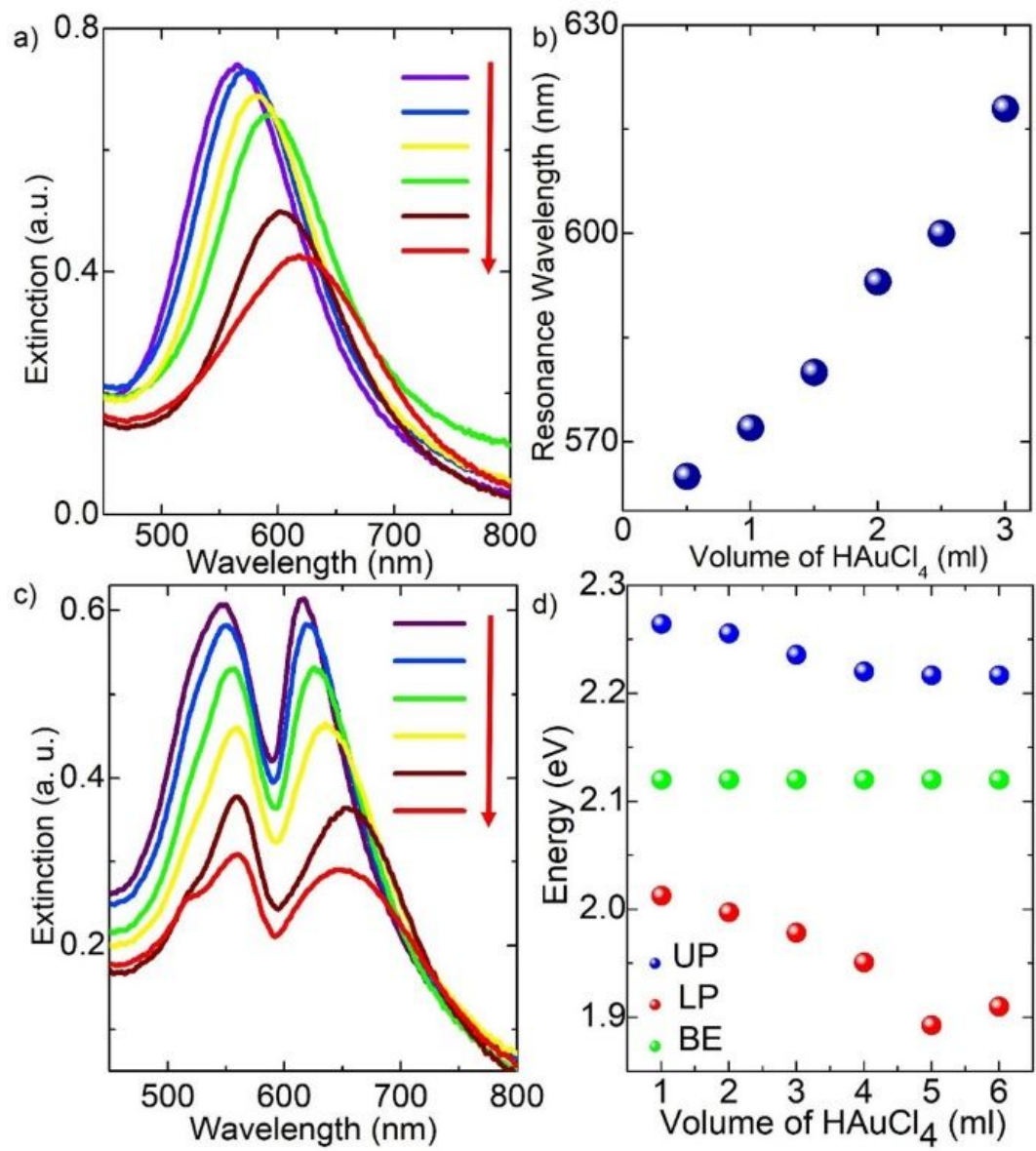

Figure S9. Colloidal nanoring shaped plexcitonic nanoparticles. (a) Experimental extinction spectra of silver nanodisks as a function of $\mathrm{HAuCl}_{4}$ volume. The curves from left to right and the red arrow correspond to the increase in $\mathrm{HAuCl}_{4}$ volume. (b) The plasmon resonance wavelength increases as a function of $\mathrm{HAuCl}_{4}$ volume. (c) The experimental extinction spectra of plexcitonic nanoparticles synthesized from the plasmonic nanoparticles shown in (a). The red arrow shows the direction of the increase in $\mathrm{HAuCl}_{4}$ volume. (d) Lower and upper polariton branches of the plexcitonic nanoparticles calculated from the experimental extinction spectra shown in (c). As the volume of $\mathrm{HAuCl}_{4}$ increases, the amplitude of the transparency dip at the resonance position decreases due to the interband transitions in gold. Plexcitonic nanorings were synthesized by self-assembly of J-aggregate dyes on bimetallic nanorings. Firstly, nanorings having plasmon resonance wavelengths ranging from $570 \mathrm{~nm}$ to $630 \mathrm{~nm}$ were synthesized by increasing the amount of $\mathrm{HAuCl}_{4}$ added to the silver nanodisk colloid. In order 
to synthesize plexcitonic nanoparticles, the colloid was mixed with varying amounts of dye solution. It is clear that plasmon resonance increases from left to right in the Figure due to the nanoring formation. In order to synthesize plexcitonic nanoparticles, the colloid was mixed with varying amounts of dye solution. In fact, Figure S9c shows extinction spectra of the hybrid nanoparticles. The calculated lower and upper polariton branches of the plexcitons from Figure S9c is displaced in Figure S9d. In the strong coupling regime, plasmons and excitons interact strongly and consequently, new hybrid polariton modes are formed.
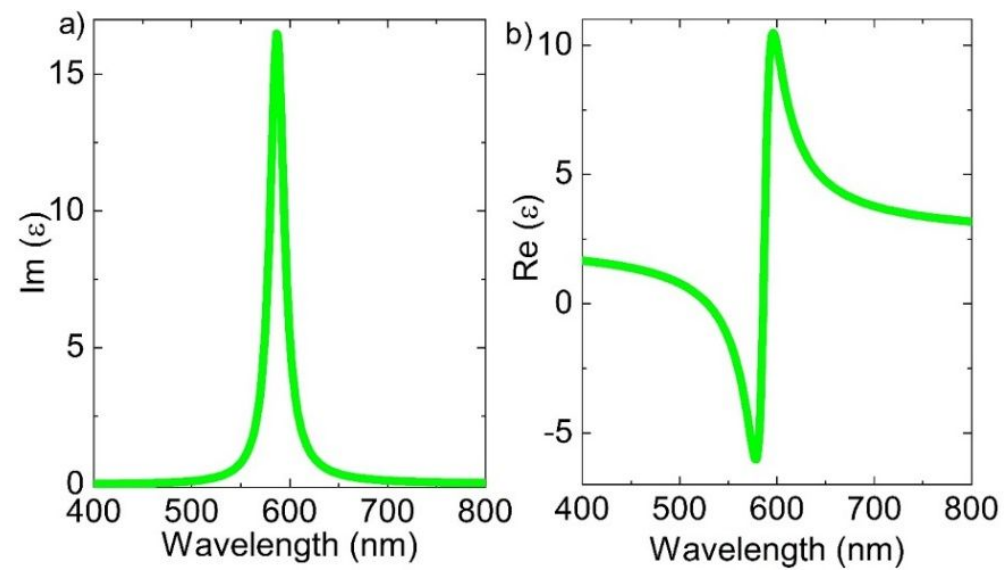

Figure S10. (a) Imaginary and (b) real parts of the dielectric function of Lorentz Oscillator used for modeling J-aggregate thin film on silver nanoparticles in theoretical calculations.

\section{References}

1. Balci, F. M.; Sarisozen, S.; Polat, N.; Balci, S., Colloidal Nanodisk Shaped Plexcitonic Nanoparticles with Large Rabi Splitting Energies. J Phys Chem C 2019, 123, 26571-26576.

2. Bek, A.; Jansen, R.; Ringler, M.; Mayilo, S.; Klar, T. A.; Feldmann, J., Fluorescence Enhancement in Hot Spots of Afm-Designed Gold Nanoparticle Sandwiches. Nano Lett 2008, 8, 485-490.

3. Wurthner, F.; Kaiser, T. E.; Saha-Moller, C. R., J-Aggregates: From Serendipitous Discovery to Supramolecular Engineering of Functional Dye Materials. Angew Chem Int Edit 2011, 50, 3376-3410.

4. Balci, S.; Kocabas, C., Ultra Hybrid Plasmonics: Strong Coupling of Plexcitons with Plasmon Polaritons. Opt Lett 2015, 40, 3424-3427.

5. Balci, S., Ultrastrong Plasmon-Exciton Coupling in Metal Nanoprisms with J-Aggregates. Opt Lett 2013, 38, 4498-4501.

6. Chikkaraddy, R.; de Nijs, B.; Benz, F.; Barrow, S. J.; Scherman, O. A.; Rosta, E.; Demetriadou, A.; Fox, P.; Hess, O.; Baumberg, J. J., Single-Molecule Strong Coupling at Room Temperature in Plasmonic Nanocavities. Nature 2016, 535, 127-130. 